\title{
Nuclear data sensitivity studies towards understanding of fuel temperature coefficient in AHWR
}

\author{
Anek Kumar ${ }^{1,2}$, Umasankari Kannan, S. Ganesan, and R. Srivenkatesan
}

Bhabha Atomic Research Centre, Mumbai-400 085, India

\begin{abstract}
The Advanced Heavy Water Reactor (AHWR) is a 920 MWth, vertical pressure tube type thorium-based reactor cooled by natural convection by boiling light water and moderated by heavy water. In this paper, we present the isotopic and energy group wise contributions to the Fuel Temperature Coefficient (FTC) in AHWR using our first order perturbation analysis code. In AHWR, the contribution of ${ }^{239} \mathrm{Pu}$ to FTC decreases with burn-up and the contribution of ${ }^{233} \mathrm{U}$ in FTC becomes more negative with burn-up. Also ${ }^{232} \mathrm{Th}$ gives a significant negative contribution, which makes the FTC become more negative with burn-up in AHWR.
\end{abstract}

\section{Introduction}

Fuel Temperature Coefficient (FTC) is an important safety parameter and a prompt component of the power coefficient of the reactor. The value of FTC depends on fissile-fertile concentrations, coolant temperature and conditions, burn-up, geometry, etc. This study of FTC in the Advanced Heavy Water Reactor (AHWR) was motivated by considerations that the estimation of FTC in a Pressurized Heavy Water Reactor (PHWR) received a special attention following the recent overpower transient event [1,2] in Kakrapar Atomic Power Station (KAPS-1, 220MWe PHWR), Kakrapar, India. In the simulation of the over-power transient incident, one of the important feedback coefficients is the FTC. The initial assessment for KAPS-1 was carried out using the feedback reactivity coefficients based on the WIMS 27 group neutron cross section library, which is a condensed version of the 69 group WIMS library of the early sixties [3]. The predicted power rise was much less steeper than the observed power rise. The various feedback reactivity coefficients were estimated [2] again using the recent WIMSD library derived from the ENDF-B/VI.8 cross section library as part the Coordinated Research Programme (CRP) of the International Atomic Energy Agency entitled, "Final Stage of the WIMSD Library Update Project (WLUP)" which ended in 2001 [4]. The feedback reactivity coefficients like the power coefficient was worked out and found to be lower than the earlier values [2]. Using the re-calculated power coefficient and taking account the operational history, this transient analysis was performed and the over-power transient was explained [2]. The KAPS-1 over-power transient could be explained [2] only with the use of new WLUP libraries [4]. In this work, we present results of nuclear data sensitivity studies in order to obtain better insight on the behaviour of FTC with burn-up in AHWR. A perturbation tool based upon WIMS conventions [3] and the new IAEA WIMSD libraries [4] was developed to perform our studies. Such an insight and understanding was also required by the Indian Atomic Energy Regulatory Board in its critical review of AHWR design. In the present

\footnotetext{
${ }^{1}$ Presenting author, e-mail: kanek@barc.gov .in

${ }^{2}$ Present address: RPDD, BARC, Mumbai, India
}

study also, we use the recent WIMSD library derived from the ENDF-B/VI.8 cross section [4].

\section{Perturbation theory}

We use first order perturbation in our approach. The objective of first order perturbation theory is to provide an estimate of $\Delta k$ without requiring a $\mathrm{k}$-calculation of the perturbed configuration (see, for example, ref. [5]).

$$
\frac{\Delta k}{k^{2}}=\frac{\sum_{g} \int\left[\begin{array}{c}
\Phi_{g}^{+} \nabla \cdot\left(\Delta D_{g} \nabla \Phi_{g}\right)-\Delta \Sigma_{0, g} \Phi_{g}^{+} \Phi_{g} \\
+\sum_{g^{\prime}} \Delta \Sigma_{s 0, g^{\prime} \rightarrow g} \Phi_{g}^{+} \Phi_{g^{\prime}}+\sum_{g^{\prime}} \frac{\Delta\left(v \Sigma_{f, g^{\prime} \rightarrow g}\right)}{k} \Phi_{g}^{+} \Phi_{g^{\prime}}
\end{array}\right] d V}{\sum_{g} \int \sum_{g^{\prime}} v \Sigma_{f, g^{\prime} \rightarrow g} \Phi_{g}^{+} \Phi_{g^{\prime}} d V}
$$

This is the form that is typically used to estimate reactivity worth using deterministic methods.

The perturbations, which are assumed to be small, are then given by

$$
\begin{gathered}
\Delta D_{g}=D_{g}^{*}-D_{g} \\
\Delta \sigma_{0, g}=\Sigma_{0, g}^{*}-\Sigma_{0, g} \\
\Delta \Sigma_{s 0, g^{\prime} \rightarrow g}=\Sigma_{s 0, g^{\prime} \rightarrow g}^{*}-\Sigma_{s 0, g^{\prime} \rightarrow g} \\
\Delta\left(v \Sigma_{f, g^{\prime} \rightarrow g}\right)=v \Sigma_{f, g^{\prime} \rightarrow g}^{*}-v \Sigma_{f, g^{\prime} \rightarrow g} . \\
\Delta k=k^{*}-k .
\end{gathered}
$$

Here $D_{g}, \Sigma_{0, g}, \Sigma_{s 0, g^{\prime} \rightarrow g}, v \Sigma_{f, g \rightarrow g}$ are the diffusion constant, total cross section, group to group scattering cross section and nu $\times$ fission cross section respectively for the unperturbed system. Other symbols represent respectively the corresponding quantities for the perturbed system.

\section{Method adopted in calculation}

Using the WIMSD code [3] we perform the AHWR lattice cell calculations. We obtain the number density and fluxes region wise in the lattice from the output of the WIMS code. 
We then obtain the cell averaged parameters using region averaged parameters using the expression, mentioned below

$$
Q_{C}=\frac{\sum_{R} Q_{R} \Phi_{R} V_{R}}{\sum_{R} \Phi_{R} V_{R}},
$$

where

$Q_{R}$ is region averaged parameter

$\Phi_{R}$ is region averaged flux

$V_{R}$ is volume of the region

$Q_{C}$ is cell averaged parameter.

Suppose that due to some perturbation there is a change in a given region averaged parameter, then accordingly one can find out the change in cell averaged parameter using the equation (2).

Region averaged parameters are calculated independently in the perturbation code using the same procedure as used in WIMS. In the perturbation code, group averaged lattice cross sections for each isotope are calculated for a given temperature and dilution cross section. To calculate the quantity "nu $\times$ sigmaf" and "absorption" cross section in the thermal region $1 / \sqrt{T}$ interpolation is used. Here $T$ is the temperature of the region. For calculation of the scattering cross section at a desired temperature, the linear interpolation is used between scattering cross section and the temperature.

\subsection{Interpolation of resonance integral}

In WIMS-D library the resonance integral (RI) data for "absorption" and "nu $\times$ fission" parameters are given for several nuclides exhibiting resonant behavior. The $\sqrt{T}$ behaviour is only used to interpolate in exact RI values per resonance group, so it can also account for group-to-group variations of the Doppler effect. For the interpolation in $\sigma_{b}$, the method of Segev is used.

\subsubsection{Calculation of the dilution parameter $\sigma_{b}$}

The perturbation code written at BARC computes the background cross section $\sigma_{b}$ for a wide range of mixtures and fuel geometries.

$$
\sigma_{b i}=\frac{1}{n_{i}}\left\{\sum_{j \neq i} n_{j} \lambda_{j} \sigma_{p j}+\sigma_{e}\right\} .
$$

Here, $n_{j}$ is the number density for the material $j$ with potential scattering cross section $\sigma_{p j}$, and $\sigma_{e}$ can be considered as the macroscopic escape cross section of the fuel (which takes care of the geometry of the fuel). In this code escape cross section $\sigma_{e}$ is taken equivalent to $a / l$ where, $a$ is the bell factor and $l$ is the mean chord length of the average fuel pin. Denoting the fuel volume by $V$ and the fuel surface by $S, l$ is the $4 S / V$. In any case, to interpolate in RI tables, $\sigma_{e}$ must be constant.

\subsubsection{Segev interpolation scheme}

This method is used for the interpolation of RI in $\sigma_{b}$. It interpolates using tabulation of $\mathrm{R}$ values, which are the RIs divided by the group width $\Delta u$, according to

$$
f(\sigma)=\frac{R(\sigma)}{R_{\infty}}=\left(\frac{\sigma}{\sigma+\eta}\right)^{p} .
$$

In equation (4), $R_{\infty}=R(\infty)$, while the constants $p$ and $\eta$ have to be determined from the two entries $R_{1}=R\left(\sigma_{1}\right)$ and $R_{2}=$ $R\left(\sigma_{2}\right)$, where $\sigma_{1}<\sigma_{2}$ so that $R_{1}<R_{2}<R_{\infty}$. If any of the latter inequalities is not true by better that $1 \%, p$ is set to 1 and $\eta$ is solved from equation (4) using only $R_{1}$.

\section{Description of AHWR lattice}

The AHWR programme is part of India's innovative efforts in advanced reactor designs [6,7]. The composite cluster analysed used in our calculations consists of a circular array of 54 fuel pins [8]. The fuel assembly has a central multi-purpose displacer rod assembly of $38 \mathrm{~mm}$ OD containing zirconium oxide. It also contains a $16 \mathrm{~mm}$ (ID) tube with radial holes for directing ECCS water injection to the fuel pins. The inner and intermediate array of 12 and 18 pins contain (Th, $\left.{ }^{233} \mathrm{U}\right)$ MOX and the outer 24 pins contain (Th, Pu) MOX. The innermost array of 12 pins has a ${ }^{233} \mathrm{U}$ content of $3.0 \%$ by weight and the middle 18 pins have $3.75 \%{ }^{233} \mathrm{U}$. The outer array of $(\mathrm{Th}, \mathrm{Pu}) \mathrm{MOX}$ pins have average of $3.25 \%$ by weight of total plutonium. The lower half of the active fuel will have $4.0 \% \mathrm{Pu}$ and the upper part will have 2.5\% Pu D5 lattice D5 lattice consisting of 54 fuel pins placed in three arrays of 12 , 18 and 24 pins in each array and a central displacer region.

Fuel composition:

Ring 1 - (Th,U)MOX - 3.0\% ${ }^{233} \mathrm{U}$ (12 pins)

Ring 2 - (Th,U)MOX - $3.75 \%{ }^{233} \mathrm{U}$ (18 pins)

Ring 3 - (Th,Pu)MOX - 3.25\% Pu (average) (24 pins)

(Lower) $4.0 \% \mathrm{Pu}$

(Upper) $2.5 \% \mathrm{Pu}$

Displacer region - $(\mathrm{ZrO} 2)$

$\mathrm{Pu}$ composition: Discharge composition of $\mathrm{Pu}$ from Indian PHWR at $6700 \mathrm{MWd} / \mathrm{T}$.

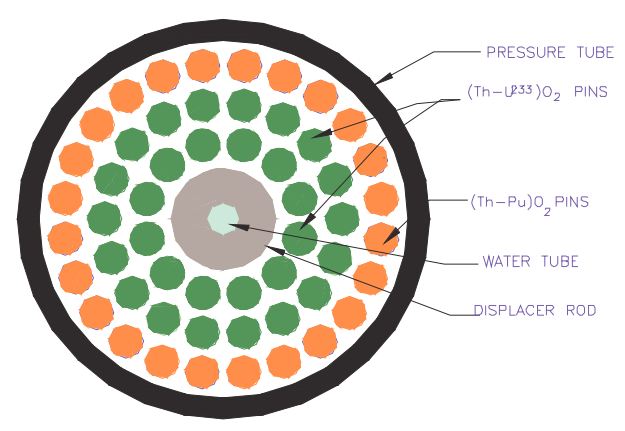

Fig. 1. Cross section of AHWR fuel cluster. 


\section{Results and discussion}

In order to estimate the fuel temperature coefficient in AHWR lattice $(4.0 \% \mathrm{Pu})$, the fuel temperature of all fuel pins was increased uniformly in the three rings. The fuel temperature was varied from $558 \mathrm{~K}$ to $723 \mathrm{~K}$. We have estimated the difference in reactivity (in $\mathrm{mk}$ ) due to different types of reaction from every isotope when temperature of the fuel goes from one value to another. In figure 2 , we present isotope wise reactivity change (in $\mathrm{mk}$ ) due to "nu×sigmaf" parameter for important isotopes in AHWR lattice when temperature of the fuel changes from $558 \mathrm{~K}$ to $723 \mathrm{~K}$. Major contribution due to perturbation ( $558 \mathrm{~K}$ to $723 \mathrm{~K}$ ) in "nu $\times$ sigmaf" parameter contributing the FTC in AHWR lattice is coming from ${ }^{233} \mathrm{U}$, ${ }^{239} \mathrm{Pu}$ and ${ }^{241} \mathrm{Pu}$ isotopes.

As we know, the FTC has two components, the Doppler and the re-thermalization coefficient. The net change due to "nu $\times$ sigmaf" parameter which is the sum of the contribution from all isotopes is found to be negative and remains negative with burn-up in AHWR case that was studied. In order to get more insight in re-thermalization coefficient, we have explicitly generated Maxwellian averaged one group cross section for these isotopes [9].

The re-thermalization component of FTC in fissile isotopes in AHWR is found to be negative, as Maxwellian averaged fission cross sections effectively is found to decrease with increasing temperature, except for ${ }^{239} \mathrm{Pu}$. We have found that for ${ }^{239} \mathrm{Pu}$ isotope, the Maxwellian averaged one group cross section increases with temperature due to the existence of $0.293 \mathrm{eV}$ resonance. For all other fissile isotopes it decreases with temperature as the fission cross section shows a nearly $1 / v$ behaviour. We can say clearly that re-thermalization component is dominating over Doppler effect in the AHWR lattice. Note also that the Pu gets incinerated fast in AHWR explaining the net decrease of the contribution of $\mathrm{Pu}$ to the FTC with burn-up.

The isotope wise scattering contribution in reactivity from important isotopes in AHWR lattice is presented in figure 3 graphically. Main contribution due to scattering reaction is coming from the ${ }^{1} \mathrm{H},{ }^{16} \mathrm{O}$ and ${ }^{2} \mathrm{D}$ isotopes. The net change

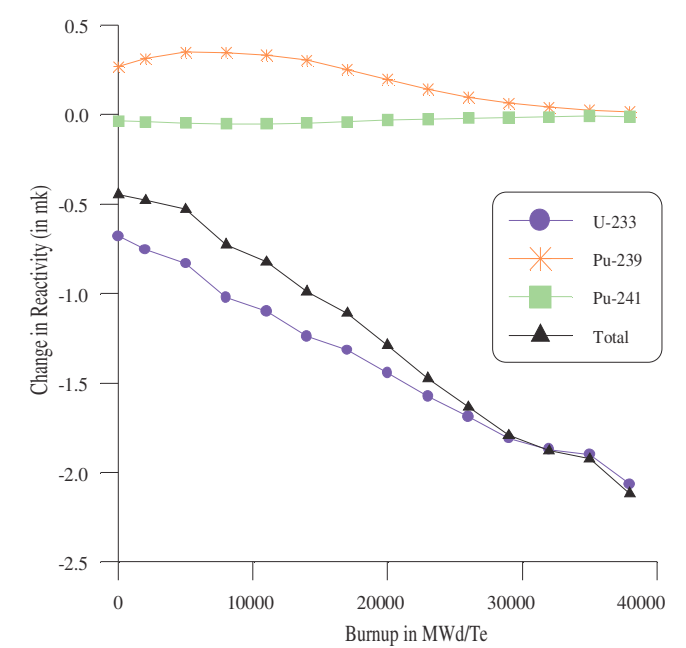

Fig. 2. Isotope wise reactivity change due to "nu $\times$ sigmaf" parameter.

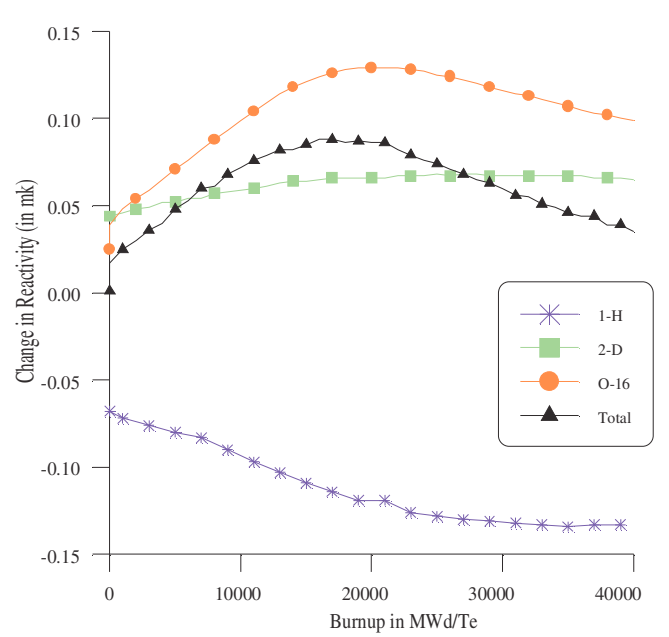

Fig. 3. Isotope wise reactivity change due to scattering reaction.

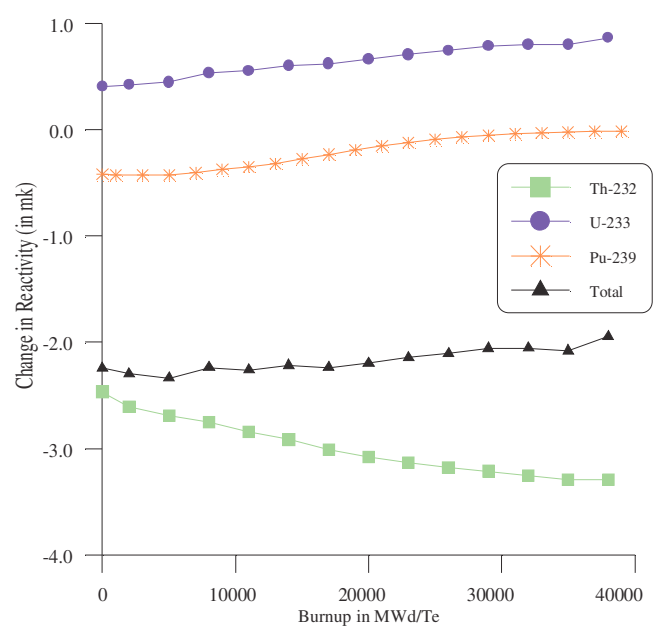

Fig. 4. Isotope wise reactivity change due to absorption reaction.

due to scattering reaction which is the sum of the contribution from all isotopes is positive and remains positive with burnup. It may be noted that in the case of PHWR, the recent BARC studies also clearly demonstrated [10] that the positive component of the FTC in natural $\mathrm{UO}_{2}$ fuelled PHWRs arises primarily due to the temperature dependence of scattering cross sections of ${ }^{16} \mathrm{O}$ in agreement with the earlier findings of Rudi Stammler [11].

The temperature of the coolant is not changed in our FTC calculations. We note that the flux in all energy groups in the coolant is reduced when temperature of the fuel changes from $558 \mathrm{~K}$ to $723 \mathrm{~K}$ due to increased absorptions in the fuel which makes the contribution in reactivity from ${ }^{1} \mathrm{H}$ to the FTC negative.

In moderator the neutron spectrum becomes harder, and flux in fast region increases due to fuel temperature rise. Accordingly the scattering contribution to FTC from ${ }^{2} \mathrm{D}$ isotope is positive. Since ${ }^{16} \mathrm{O}$ is present in fuel, the scattering cross section of ${ }^{16} \mathrm{O}$ increases as temperature of the fuel increases. This makes the scattering contribution from ${ }^{16} \mathrm{O}$ to FTC positive in lattice.

In figure 4 , we present isotope wise reactivity change (in $\mathrm{mk}$ ) due to absorption reaction parameter for important isotopes in AHWR lattice when temperature of the fuel changes 
from $558 \mathrm{~K}$ to $723 \mathrm{~K}$. Main contribution due to absorption reaction is coming from the ${ }^{232} \mathrm{Th},{ }^{233} \mathrm{U}$ and ${ }^{239} \mathrm{Pu}$ isotopes. The net change due to absorption reaction which is the sum of the contribution from all isotopes is negative and remains negative with burn-up.

\section{Conclusion}

We obtained isotopic and energy group wise contribution in Fuel Temperature Coefficient (FTC) in AHWR using a first order perturbation analysis code. We found that in the AHWR lattice, the contribution to FTC from ${ }^{239} \mathrm{Pu}$ is very small and insensitive to the temperature variation. The temperature difference causes more significant variations in the ${ }^{232} \mathrm{Th}$ and ${ }^{233}$ U absorption reaction. For the contribution due to "sigmaf" parameter to FTC, the re-thermalization component dominates over the Doppler component. In the beginning and at low burnup, the FTC is dominated by ${ }^{239} \mathrm{Pu},{ }^{233} \mathrm{U}$ and ${ }^{232} \mathrm{Th}$. At high burn-up this is dominated by ${ }^{232} \mathrm{Th}$ and ${ }^{233} \mathrm{U}$ rather than that of ${ }^{239} \mathrm{Pu}$. Thus the FTC becomes more negative with burn-up in AHWR. This is in contrast to the behaviour of FTC in PHWR where the FTC becomes positive after a certain burn-up.

\section{References}

1. S. Ganesan, New reactor concepts and new nuclear data needed to develop them, invited talk, ND2004, Santa Fe, New Mexico, American Institute of Physics (AIP) Conference Proceedings 769, May 2005, pp. 1411-1416.
2. B. Singh, S. Ganesan, P.D. Krishnani, R. Srivenkatesan, A.N. Kumar, M.V. Parikh, H.P. Rammohan, S. Ray, M.P. Fernando, S.S. Bajaj, Analysis of Power Rise Transient in KAPS-1 and Power Co-efficient Evaluation, IAEA International Conference on Operational Safety performance in Nuclear Installations, Vienna, 30 Nov.-2 Dec. 2005.

3. J.R. Askew, F.J. Fayers, P.B. Kemshell, A general description of the lattice code WIMS, British Nuclear Energy Society, Oct. 1966, p. 564.

4. WIMS-D Library Update Project, International Atomic Energy Agency, 2006, http://www-nds.indcentre.org.in/wimsd/.

5. G. Bell, S. Glasstone, Nuclear Reactor Theory (Van Nostrand Reinhold Company, 1970), pp. 273-283.

6. D. Saha, R.K. Sinha, Indian Advanced Nuclear Reactors, pp. 75-83, Proceedings, Invited talks, 6th Annual Conference of Indian Nuclear Society, INSAC-2005, edited by S. Ganesan et al., Nov. 15-18, 2005, Mumbai.

7. R.K. Sinha, A. Kakodkar, Nucl. Eng. Design 236, 683 (2006).

8. Arvind Kumar, A new cluster design for the reduction of void reactivity in $A H W R$, poster paper presented in the Indian Nuclear Society Annual Conference, INSAC-2000, June 2000.

9. Anek Kumar, S. Ganesan, Variation of Maxwellian averaged cross sections of ${ }^{232} \mathrm{Th}^{233} \mathrm{U},{ }^{235} \mathrm{U},{ }^{239} \mathrm{Pu}$ and ${ }^{240} \mathrm{Pu}$ with temperature (2006) (unpublished).

10. U. Kannan, S. Ganesan, Isotopic and Energy Groupwise Dependence of Fuel Temperature Coefficient of Reactivity in Natural Uranium Fuelled PHWRs, Nucl. Sci. Eng. (2007) (in press).

11. R.J. Stammler, Thermal contribution to Fuel-Temperature Coefficient in Water-moderated Power-Lattice Cells, Nukleonik 12, 53 (1969). 